\title{
GENDER DIFFERENCES OF COGNITIVE AND MOTIVATIONAL PREDICTORS OF STUDENT'S PSYCHOLOGICAL WELL-BEING
}

This work was supported by the RA Science Committee and Russian Foundation for Basic Research (RF) in the frames of the joint research project SCS 20RF-164 and RFBR 20-513-05014 accordingly.

Serobyan A. K., Grigoryan A. K., Khachatryan N. G. (Yerevan State University,

Yerevan, Armenia)

Yerofeeva V. G. (HSE, Moscow, Russia) astghiks@ysu.am,ani_grigoryan@ysu.am,n_khachatryan@ysu.am, yerofeyeva.victoria@yandex.ru 乙tinцшјшgर्üи uर्u. 03.08.2021

૧pupunuर्ume uर्u. 14.08.2021

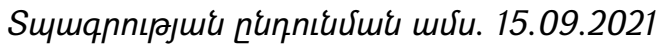

The purpose of this study was to investigate gender differences in cognitive and motivational factors of achieving success-predetermining student's psychological well-being. The Warwick-Edinburgh Mental Well-Being scale (WEMWBS) was used to measure psychological well-being.

The mindset and promotion/prevention regulatory focus were considered as cognitive and motivational predictors. The study was based upon a sample of 415 students (Mage $=20.4$, SDage $=2.568$ ), of whom 63.5\% were women. Two regression models were developed separately, for men and women to identify gender differences in factors contributing to psychological well-being. The promotion focus was a significant predictor for men's psychological well-being. Women's psychological well-being was determined by growth mindset and promotion focus. The prevention focus negatively predicted women's psychological well-being. The results of this study replicated the findings of other studies, that promotion focus has a predictive role in psychological well-being, at the same time revealing gender differences in cognitive and motivational factors of achieving success.

Keywords: Psychological well-being, mindset, self-regulatory focus, regression model.

DOI: https://doi.org/10.46991/SBMP/2022.5.1.107

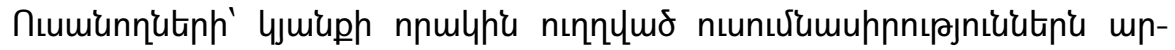

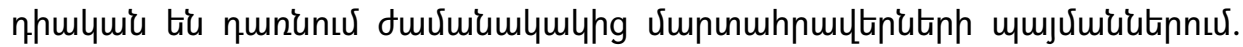

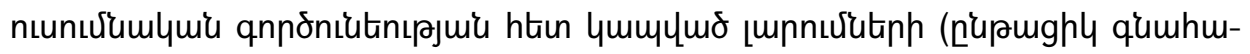

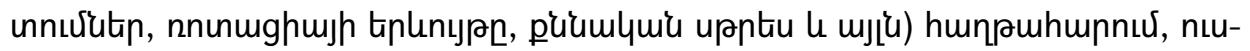




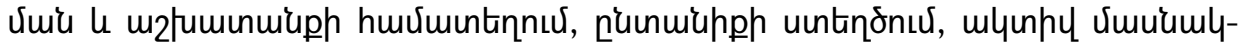

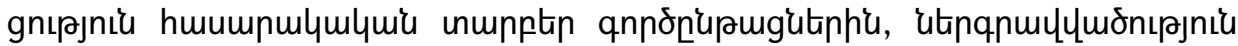

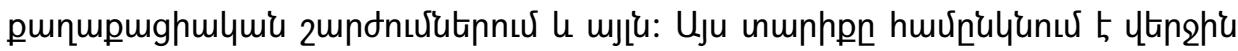

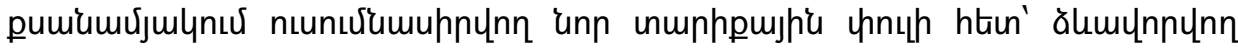

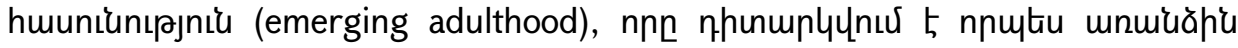

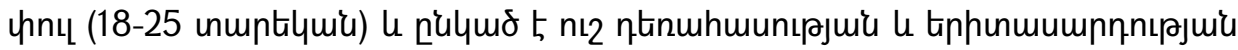

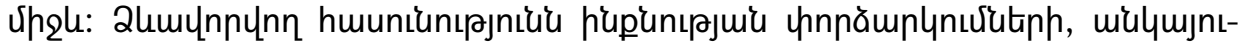

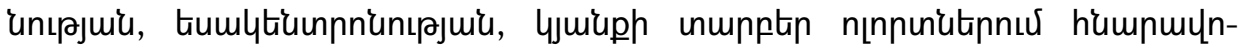

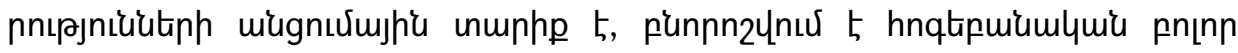

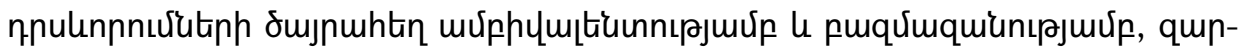

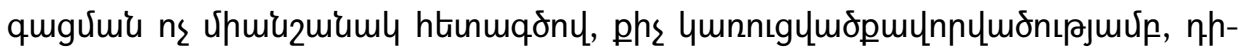

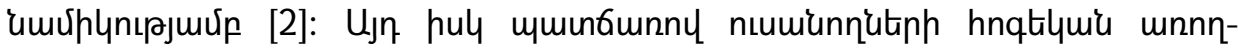

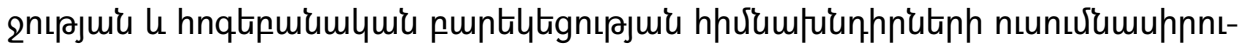

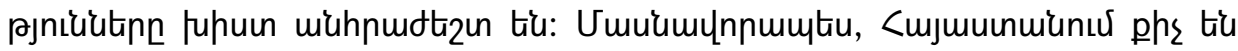

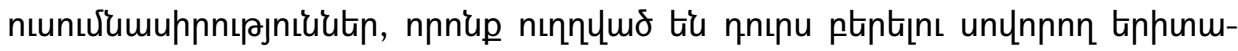

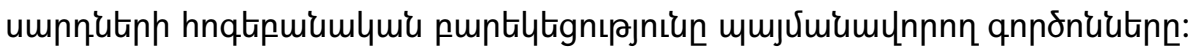

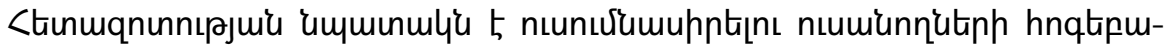

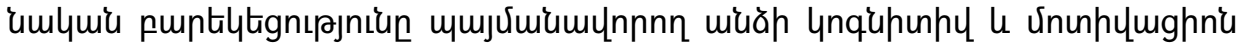

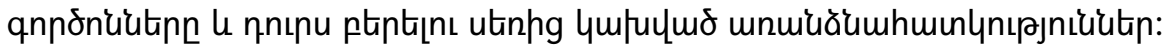

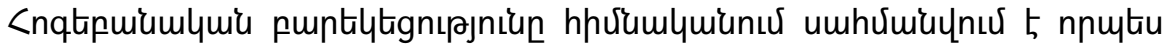

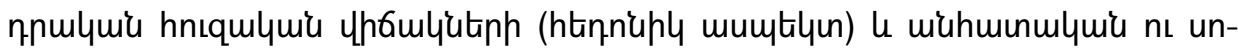

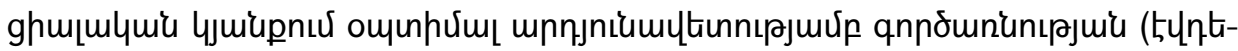

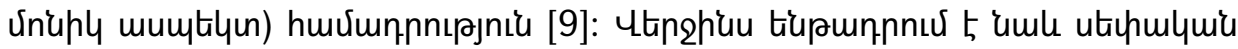

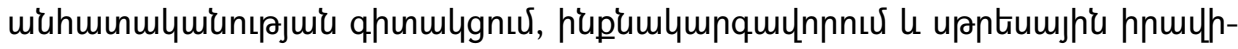

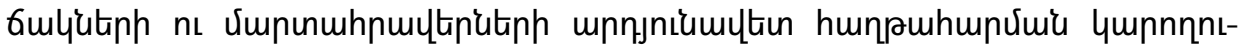

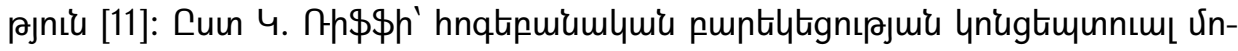

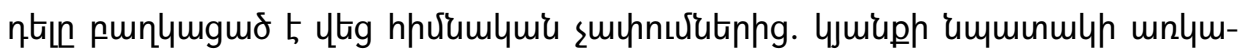

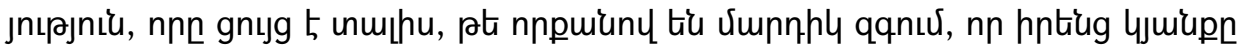

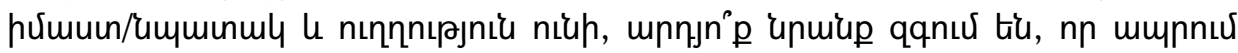

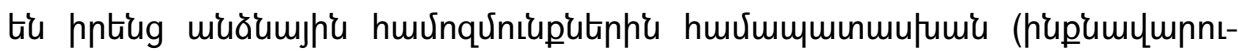

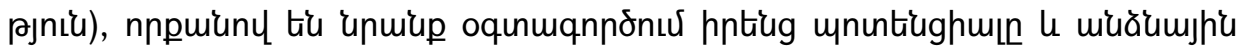

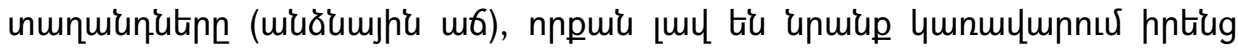

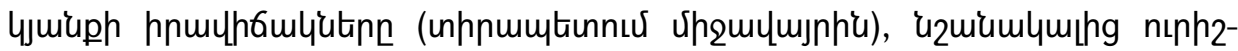

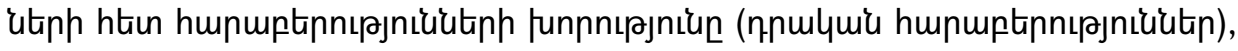

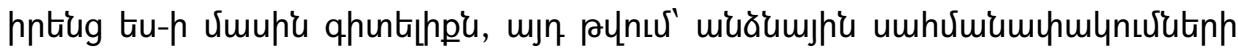

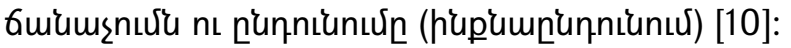

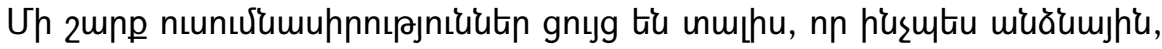

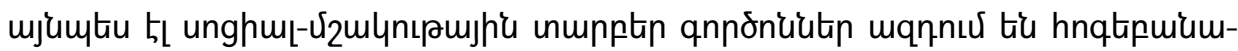

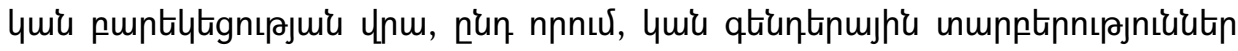

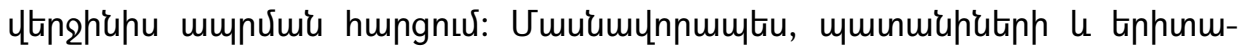




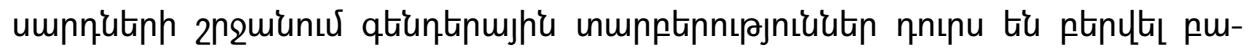

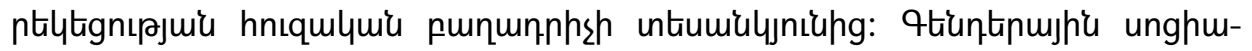

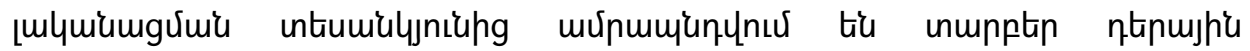

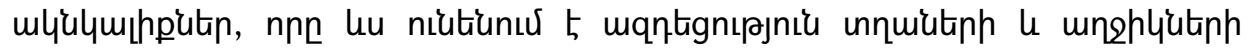
puntiltignıрјuí unu [5]:

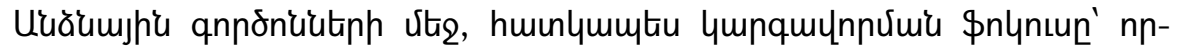

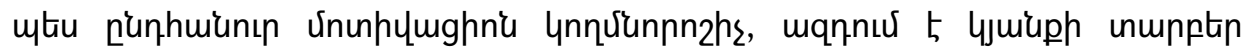

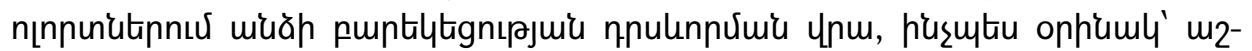

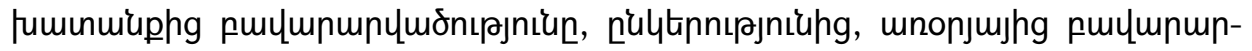

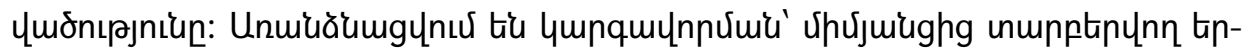

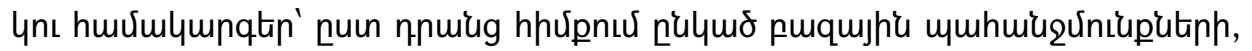

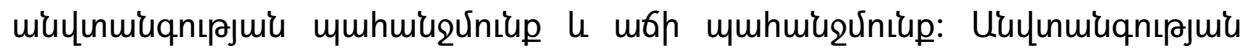

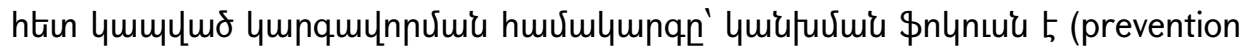

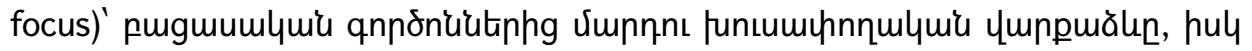

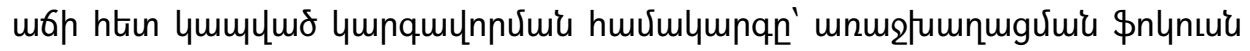

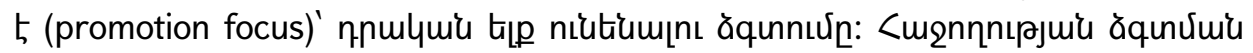

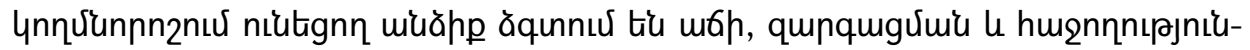

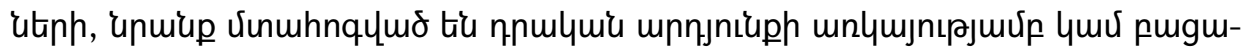

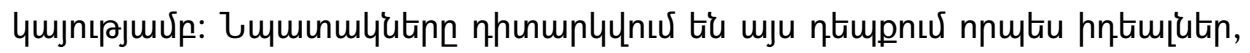

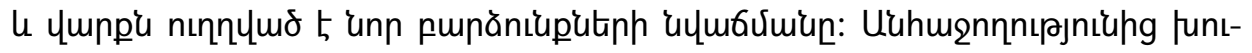

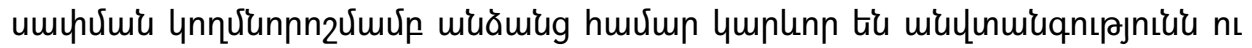

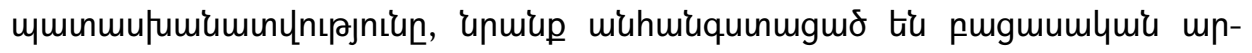

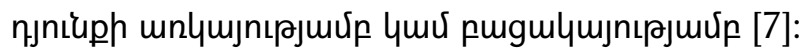

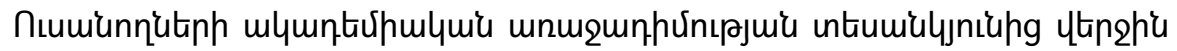

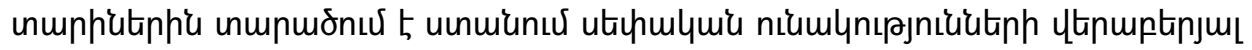

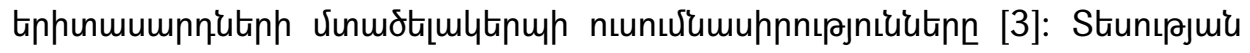

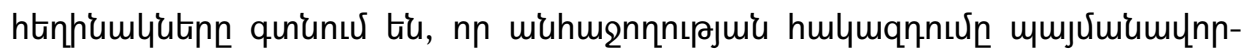

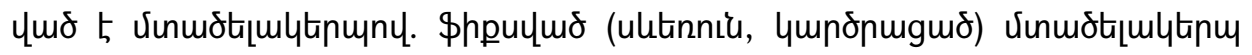

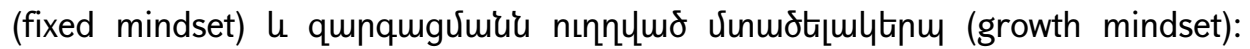

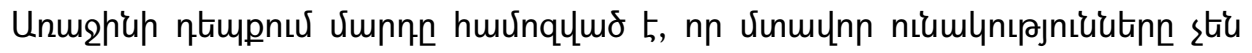

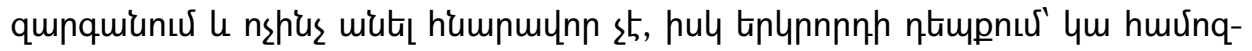

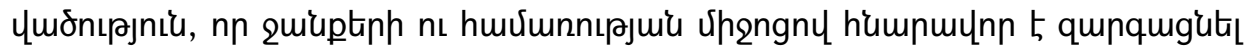

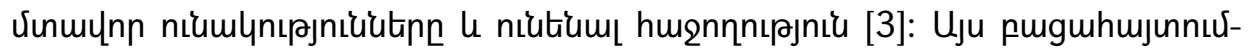

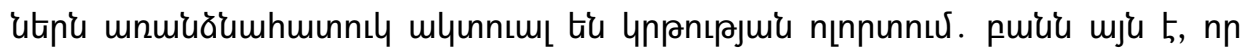

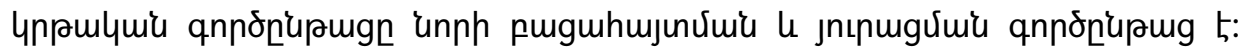

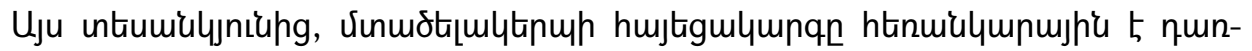

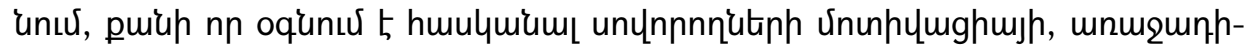

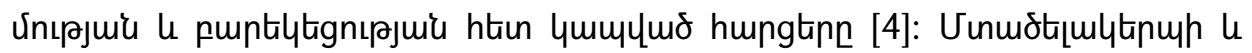

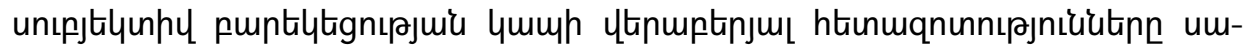

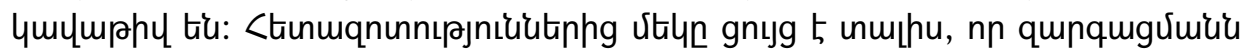




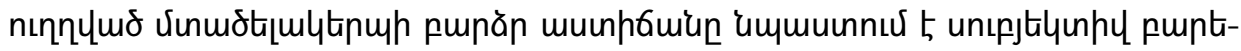

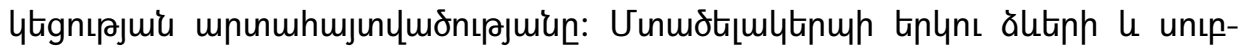

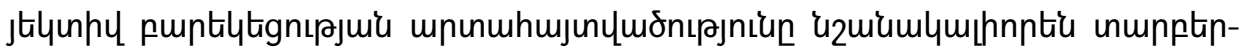

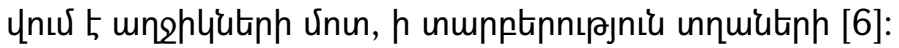

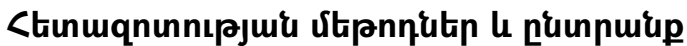

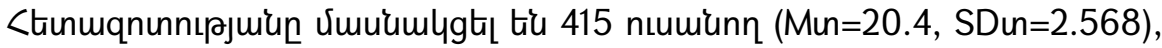

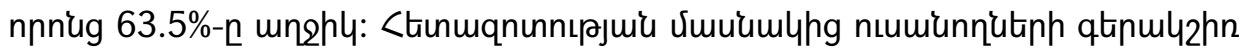

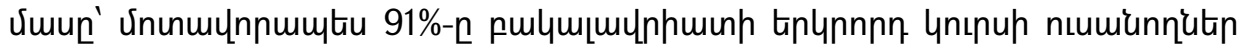

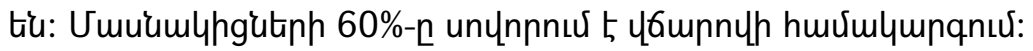

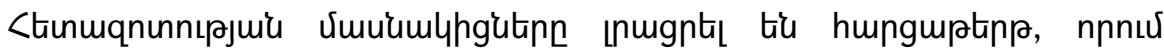

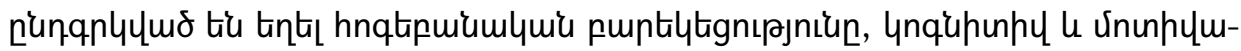

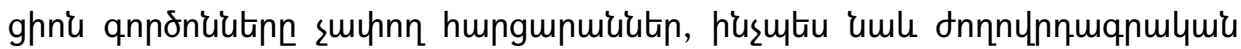

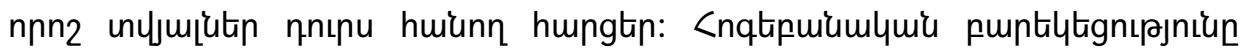

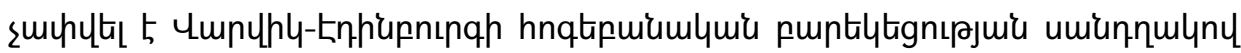
(Warwick-Edinburgh Mental Well-Being Scale' WEMWBS) [11]: Uminnnuln

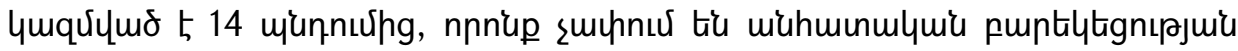

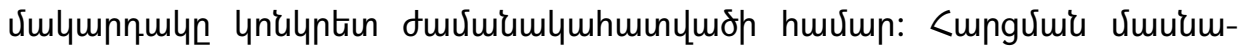

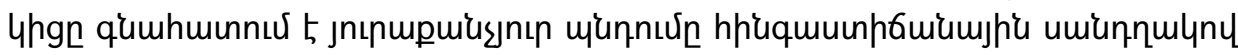

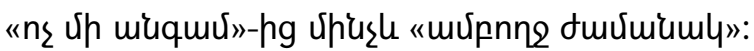

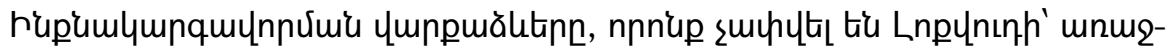

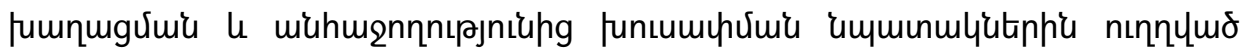

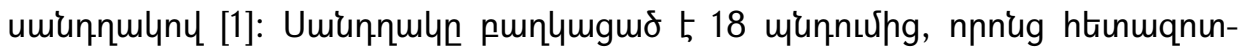

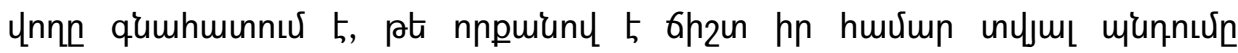

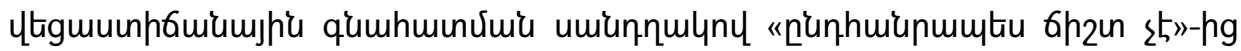

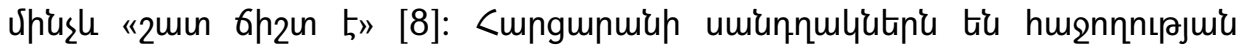

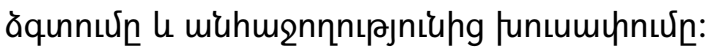

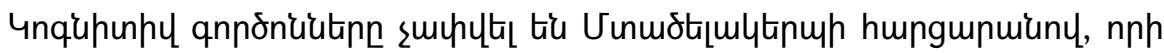

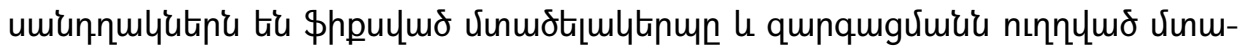

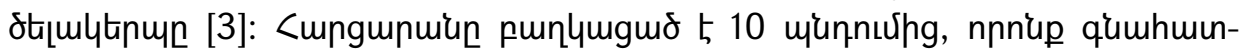

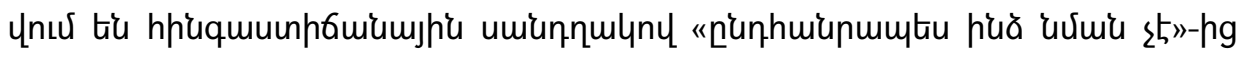

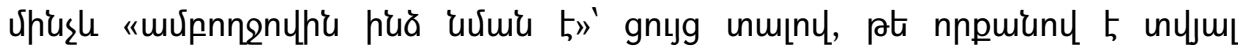

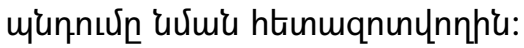

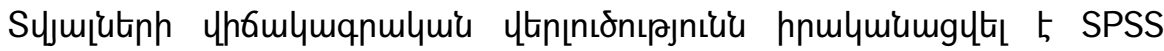
Statistics, Version 23.0 onmqnny:

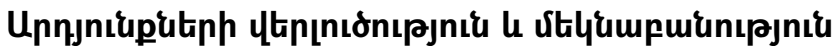

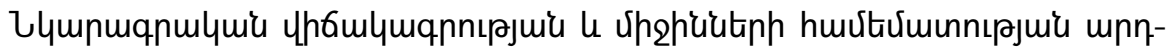

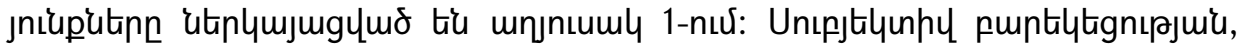

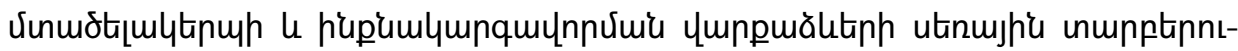

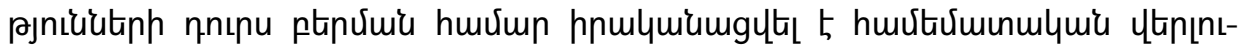

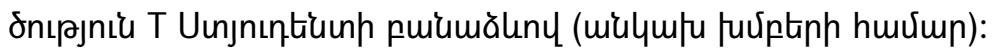




\section{Unjniuml 1.}

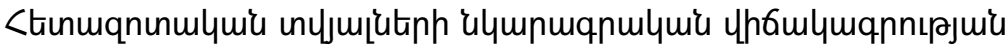

\begin{tabular}{|c|c|c|c|c|c|}
\hline \multicolumn{6}{|c|}{ upnjлtuputin } \\
\hline & 疋 & 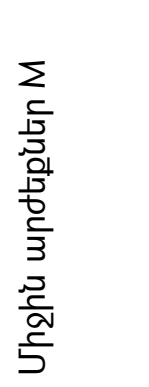 & 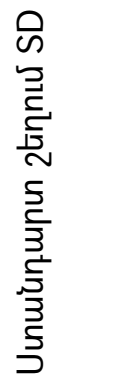 & $\begin{array}{l}\sigma \\
3 \\
3 \\
\frac{9}{3} \\
\frac{1}{2} \\
\frac{c}{2} \\
\frac{3}{3} \\
\frac{d}{3} \\
\frac{5}{5} \\
\frac{5}{5}\end{array}$ & 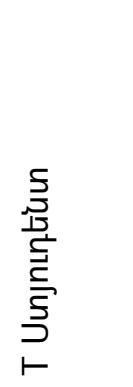 \\
\hline \multirow{2}{*}{ 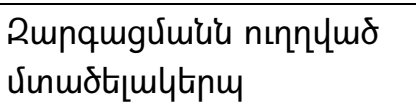 } & hq & 19.7 & 3.326 & \multirow[t]{2}{*}{0.637} & \multirow[t]{2}{*}{0.696} \\
\hline & mp & 19.4 & 3.942 & & \\
\hline \multirow[t]{2}{*}{ 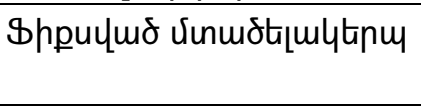 } & hq & 12.3 & 3.707 & \multirow[t]{2}{*}{0.579} & \multirow[t]{2}{*}{0.752} \\
\hline & un & 12.0 & 4.082 & & \\
\hline \multirow[t]{2}{*}{ 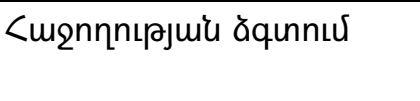 } & hq & 5.1 & 0.821 & \multirow[t]{2}{*}{0.870} & \multirow[t]{2}{*}{$-2,654^{* *}$} \\
\hline & up & 4.8 & 0.949 & & \\
\hline \multirow{2}{*}{ 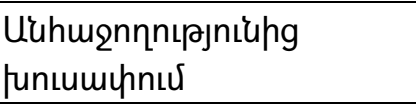 } & hq & 4.1 & 0.897 & \multirow[t]{2}{*}{0.717} & \multirow[t]{2}{*}{$-3,518^{* *}$} \\
\hline & un & 3.7 & 0.829 & & \\
\hline
\end{tabular}

$$
\mathrm{p}^{* *}<0.01
$$

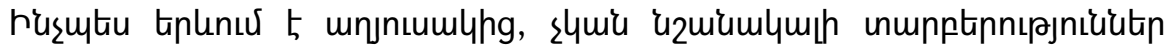

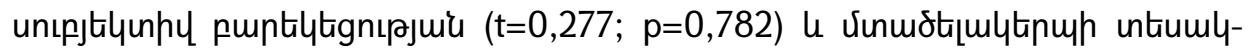

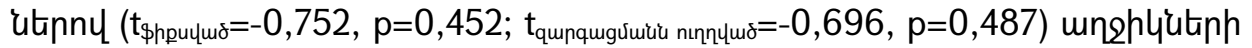

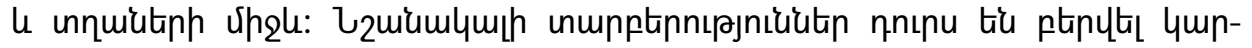

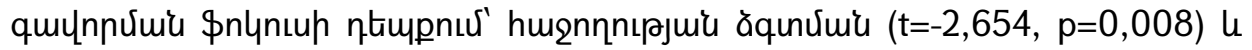

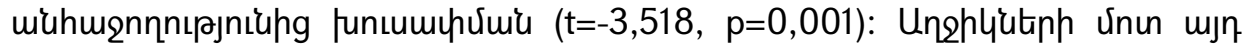

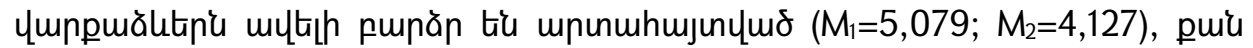
unquitiph unun $\left(M_{1}=4,779 ; M_{2}=3,718\right)$ :

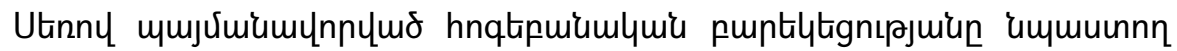

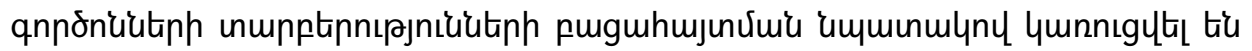

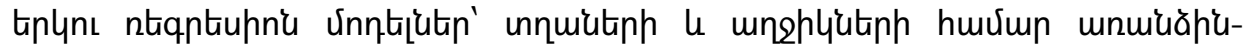

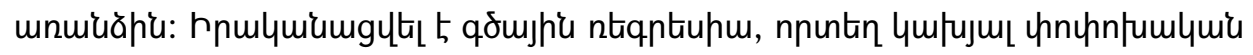

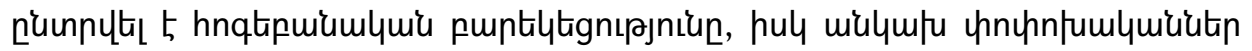

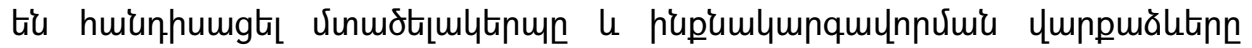

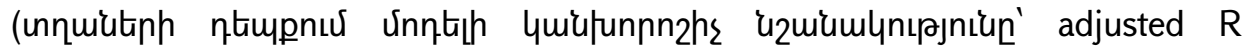

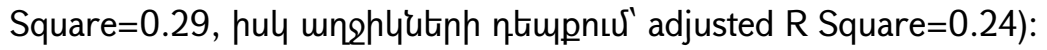

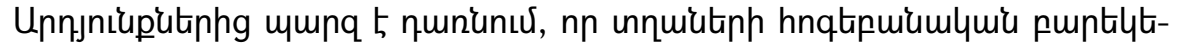

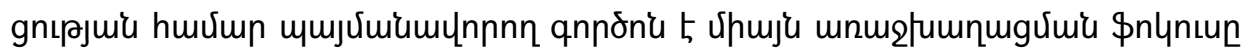

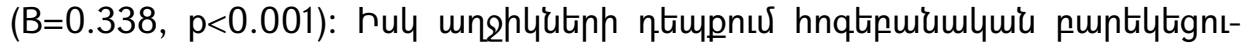




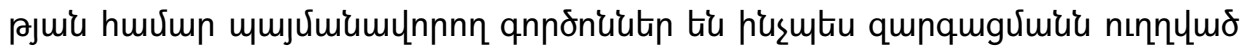

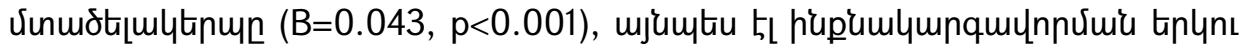

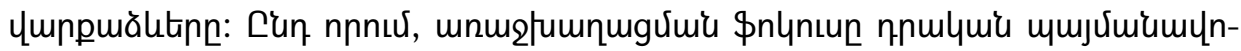

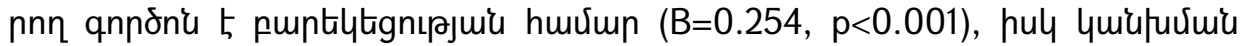
\$nlntun' pugmumumiu $(B=-0.116, p<0.05)$ :

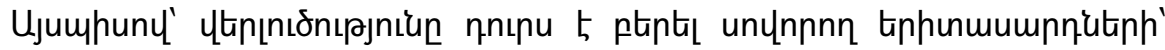

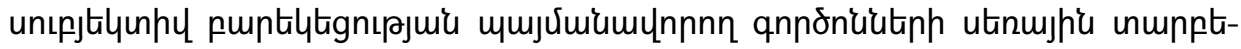

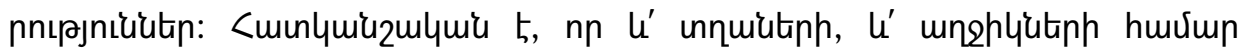

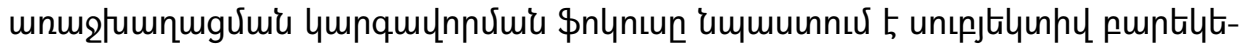

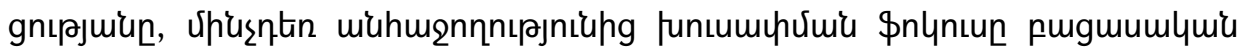

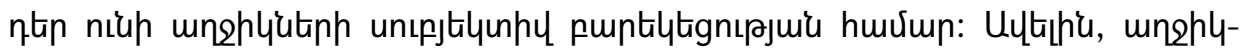

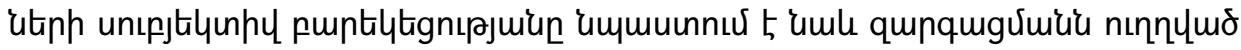

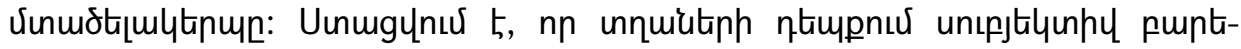

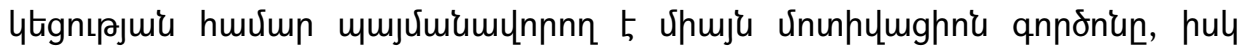

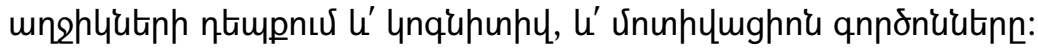

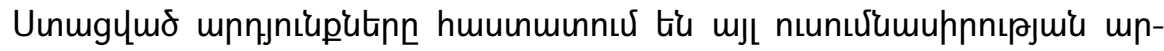

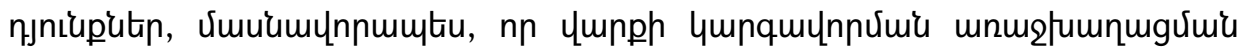

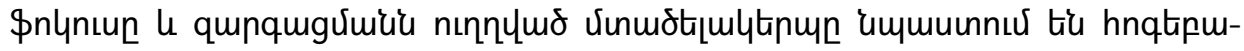

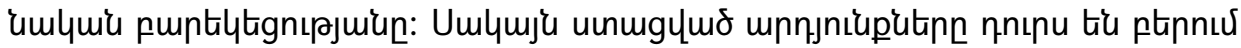

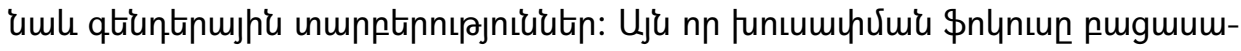

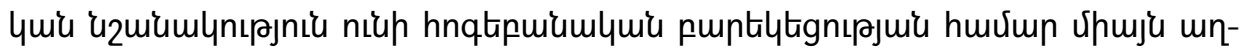

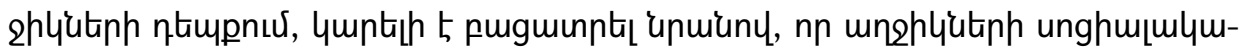

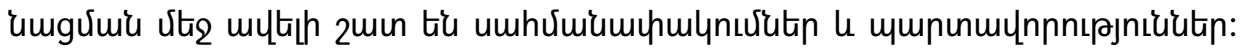

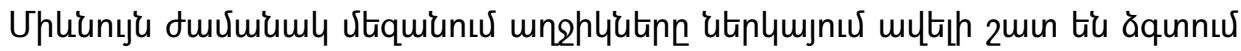

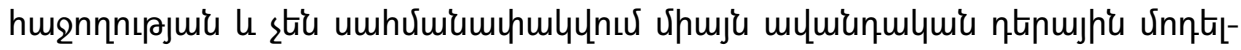

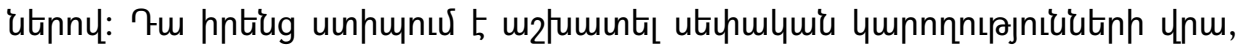

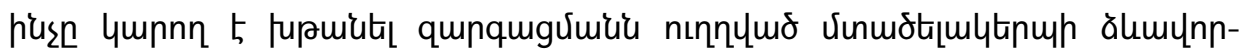

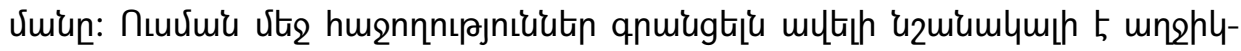

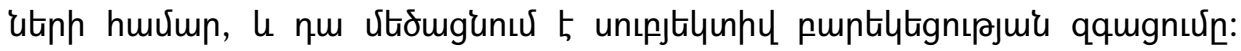

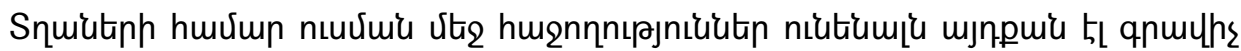

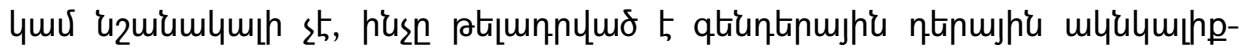

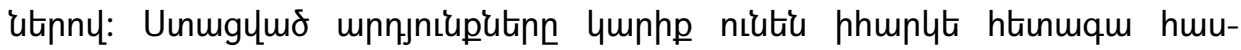

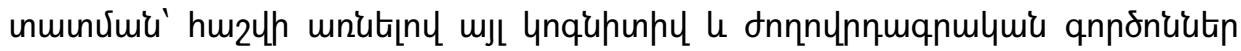

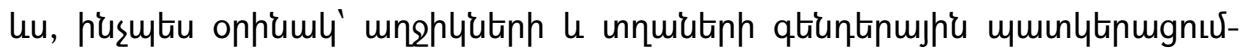

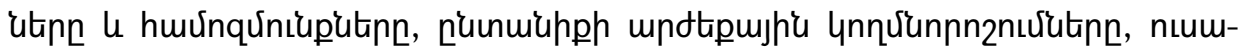

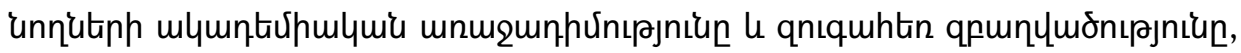

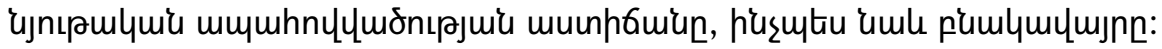




\section{9puluúnıpjnı\&}

1. Abu ELSamen, Amjad \& Mowen, John \& Fang, Xiang Examining the Construct Validity of the Lockwood Goal Orientation Scale, 2015. 10.1007/978-3-319-11797-3_11.

2. Arnett, J. J. Emerging Adulthood: What is it, and what is it good for? // Child development perspective, 1 (2), 2007, pp. 68-73

3. Dweck, C. S. Mindset: The new psychology of success. New York House Inc., 2006.

4. Dweck, Carol \& Yeager, David. Mindsets: A View from Two Eras. Perspectives on Psychological Science. 14. 2019. 174569161880416. 10.1177/1745691618804166.

5. Esteban-Gonzalo, S., Esteban-Gonzalo, L., Cabanas-Sánchez, V., Miret, M., \& Veiga, O. L. (2020). The Investigation of Gender Differences in Subjective Wellbeing in Children and Adolescents: The UP\&DOWN Study. International journal of environmental research and public health, 17(8), 2732. https://doi.org/10.3390/ijerph17082732

6. Gunnarsdóttir S. Growth vs. fixed mindset : the relationship between student's mindset, resilience and psychological well-being, http://hdl.handle.net/1946/39287

7. Higgins E. T. Value from regulatory fit. Current Directions in Psychological Science, 14, 2005, pp. 209 - 213.

8. Lockwood, P., Jordan, C. H., \& Kunda, Z. Motivation by positive or negative role models: Regulatory focus determines who will best inspire us. Journal of Personality and Social Psychology, 83(4), 2002, pp. 854864. https://doi.org/10.1037/0022-3514.83.4.854

9. Ryan RM, and Deci EL. On happiness and human potential: a review of research on hedonic and eudemonic well-being. Annual Review of Psychology, 52, 2001, pp. 141-166.

10. Ryff C. D. (2014). Psychological well-being revisited: advances in the science and practice of eudaimonia. Psychotherapy and psychosomatics, 83(1), 10-28. https://doi.org/10.1159/000353263

11. Stewart-Brown, Sarah \& Platt, Stephen \& Tennant, Alan \& Maheswaran Hendramoorthy \& Parkinson, Jane \& Weich, Scott \& Tennant, Ruth \& Taggart, Frances \& Clarke, Aileen. The WarwickEdinburgh Mental Well-being Scale (WEMWBS): A valid and reliable tool for measuring mental well-being in diverse populations and projects. Journal of Epidemiology and Community Health. 65. A38-A39. 2011. 10.1136/jech.2011.143586.86. 


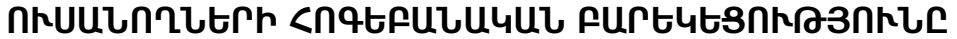

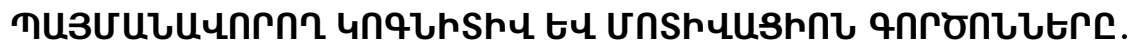

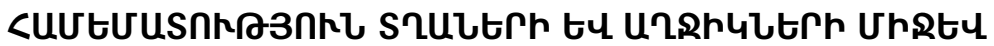

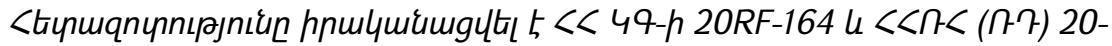

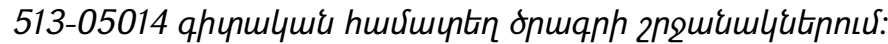

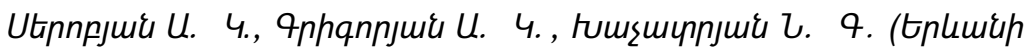

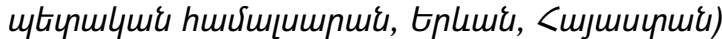

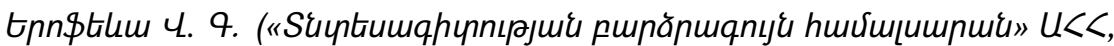

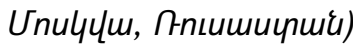

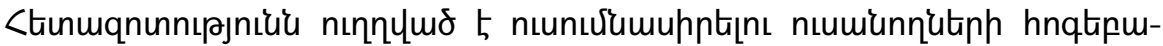

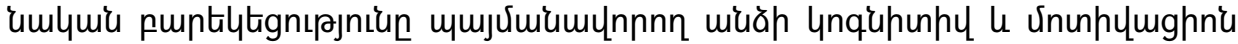

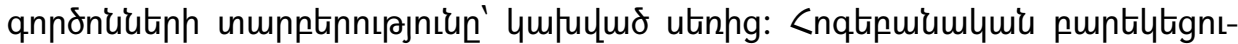

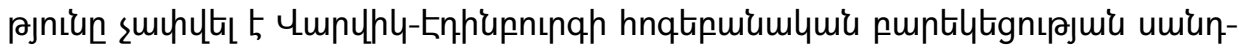
nulyny (Warwick-Edinburgh Mental Well-Being Scale' WEMWBS): กnultu hnqt-

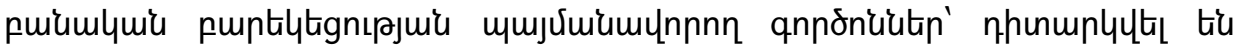

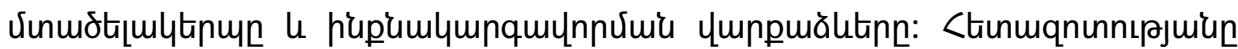
vimukumgta t 415 numiunn (Mun=20.4, SDun=2.568), nnnig 63.5\%-n ungh4:

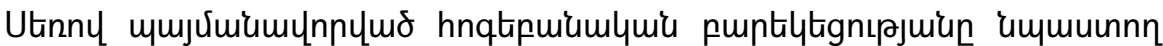

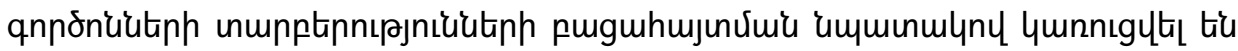

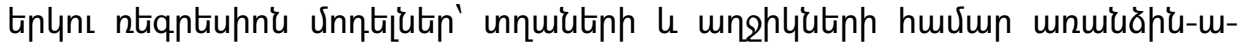

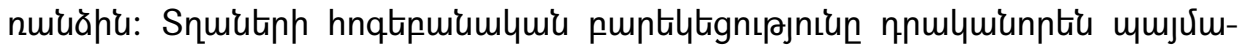

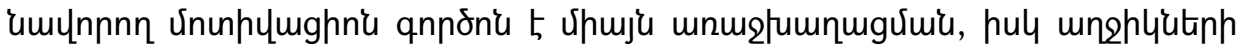

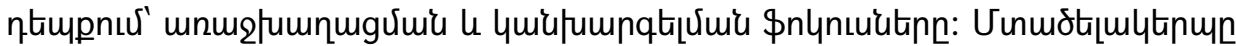

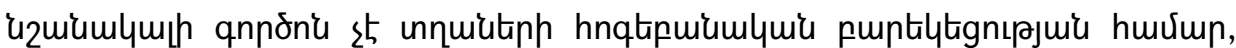

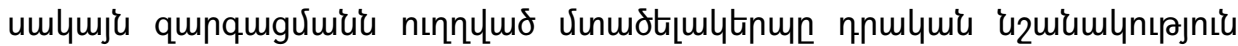

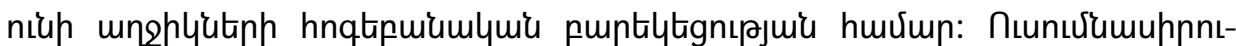

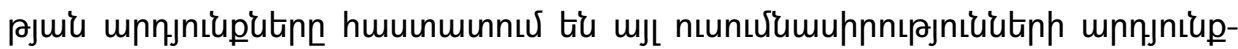

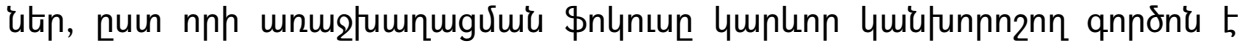

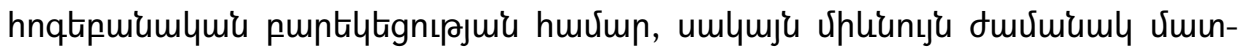

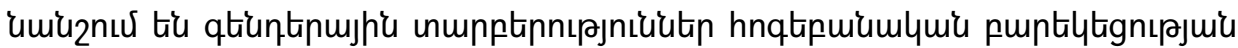
unquhunhy u ununpumghn'u qnnonuututph ưpol:

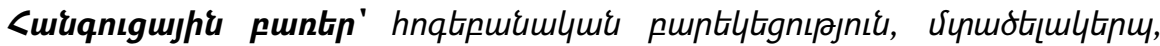

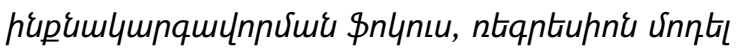




\section{ГЕНДЕРНЫЕ РАЗЛИЧИЯ КОГНИТИВНЫХ И МОТИВАЦИОННЫХ ПРЕДИКТОРОВ ПСИХОЛОГИЧЕСКОГО БЛАГОПОЛУЧИЯ СТУДЕНТОВ}

Исслед. вып. при финансовой поддержке КН РА и РФФИ (РФ) в рамках совместой научной программы 20RF-164 и 20-513-05014 соответственно. Серобян А. К., Григорян А. К., Хачатрян Н. Г. (Ереванксий государственный университет, Ереван, Армения) Ерофеева В. Г. (НИУ ВШЭ, Москва, Россия)

Целью данного исследования было изучение гендерных различий в когнитивных и мотивационных фракторах достижения успеха, предопределяющих психологическое благополучие учащихся. Для измерения психологического благополучия использовалась шкала психического благополучия УорикаЭдинбурга.

В качестве когнитивных и мотивационных предикторов рассматривался образ мышления и регуляторный фокус. Исследовательская выборка состояла из 415 студентов (Мвозр = 20,4, SDвозр = 2,568), из которых 63,5\% составляли женщины. Был проведен регрессионный анализ отдельно для мужчин и женщин для выявления гендерных различий в факторах, способствующих психологическому благополучию. Фокус продвижения был позитивным предиктором психологического благополучия у мужчин. Психологическое благополучие женщин определялось установкой на рост и фокусом продвижения. Фокус предупреждения был негативным предиктором психологического благополучия у женщин.

Результаты этого исследования подтверждают результаты других исследований, что фокус продвижениия играет прогностическую роль в психологическом благополучии, в то же время выявляя гендерные различия в когнитивных и мотивационных фракторах достижения успеха.

Ключевые слова: психологическое благополучие, образ мышления, регуляторный фокус, регрессионная модель. 